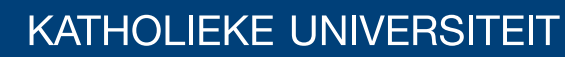 \\ LEUVEN
}

Faculty of Business and Economics

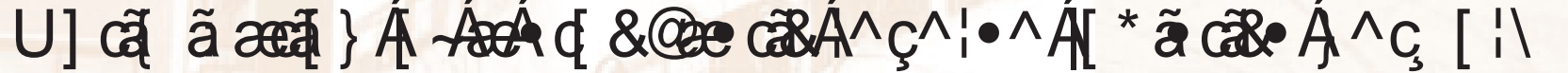
Z LKQUHXLFIMKP HQWDQGH FKDQJHIRSURQV

. UNVI IFFHQVI3 IHANU\&ROQDDQGO DIFI DP EUFFWW

DEPARTMENT OF DECISION SCIENCES AND INFORMATION MANAGEMENT (KBI) 


\title{
Optimization of a Stochastic Reverse Logistics Network with Refurbishment and Exchange Options
}

\author{
Kris T. Lieckens ${ }^{1}$, Pieter J. Colen ${ }^{1}$, Marc R. Lambrecht ${ }^{1}$ \\ ${ }^{1}$ Faculty of Business and Economics, Research Center for Operations Management \\ K.U.Leuven - Belgium \\ (kris.lieckens@econ.kuleuven.be,pieter.colen@econ.kuleuven.be,marc.lambrecht@econ.kuleuven.be)
}

\begin{abstract}
Remanufacturing activities are gaining momentum in the manufacturing industry. Therefore, the need for optimized networks becomes more pressing. In this paper we take a profit maximization approach to simultaneously determine the optimal network and the delivery strategy to support remanufacturing services offered to customers. In order to set up a network, investment decisions have to be made concerning the number, locations and types of remanufacturing facilities. Additionally, appropriate capacity and inventory levels have to be set in order to guarantee a given service level. These network decisions are influenced by the way the remanufacturing services are offered by the manufacturing firm. We consider two possible service delivery strategies: the service provider can either make a quick exchange of the used part by a refurbished one or re-install the original part after remanufacturing it. The model described in this paper is applied to optimize the network and service delivery strategy at a worldwide manufacturer of construction, mining and industrial equipment.
\end{abstract}

Keywords : Network Design, Queueing Networks, Remanufacturing, Contracting

\section{Introduction}

Original equipment manufacturers (OEMs) in many industries are facing fierce competition in their primary (new product) market. At the same time the growing installed base of equipment provides an opportunity to develop a profitable remanufacturing business. Companies such as Caterpillar and HP already have considerable remanufacturing activities and many other companies are following. The launch of more extensive warranties and overhaul services relies on remanufacturing activities and fits the current trend of more service orientation in the industrial equipment industry (Cohen et al. 2006, Pawar et al. 2009).

Now that remanufacturing activities have captured the attention of top management, they are brought to the market more actively and globally optimized reverse logistics networks are pursued. Such network decisions do not only involve the number and the location of the facilities, but also the assignment of specific tasks to appropriate facilities and determining optimal capacity and inventory levels. Furthermore, OEMs should realize that the contractual arrangements being made with customers regarding for instance the ownership of parts and the service delivery method will impact the optimal design of the reverse network. Although setting up a multi-echelon repairable parts network is a daunting task, it is a vital step in order to efficiently provide remanufacturing services. In this paper we report on our research concerning the design of a reverse logistics network. 


\section{Problem Description}

When setting up a reverse logistics network for remanufacturing services, many strategic decisions have to be taken. In this paper we take the perspective of a profit maximizing company with contractual obligations (service levels). For a logistics network to take form, clearly, a decision has to be made about the number and location of the facilities. Moreover, different types of facilities may be used to support the remanufacturing business, e.g. inspection centers or rewiring facilities. Next to the locations and types of facilities to open, the capacity levels have to be determined. Typically in the remanufacturing business, capacity will be determined by the number of operators that are employed. Furthermore, remanufacturing services can be provided through different delivery strategies. With respect to the service delivery strategy we consider two options, i.e. refurbishment without an exchange of the part and refurbishment with an exchange. In the former case, a technician travels to the customer site and disconnects the worn-out part(s). Next, the used part is sent to a remanufacturing facility. After being remanufactured, the original part returns to the owner (customer) and a technician reinstalls it. The involved machine can only be restarted when this job is completed. In the second service delivery strategy, the technician travels to the customer but already brings along a remanufactured part. The worn-out part is then exchanged by a remanufactured one, which limits the machine downtime. Next, the worn-out part is sent to a remanufacturing facility. After being remanufactured, the part can serve as an exchange part for another customer. In the first delivery strategy, the equipment downtime equals the remanufacturing lead time, whereas in the second delivery strategy the customer receives an immediate replacement.

Most research has focused on networks supporting the second delivery strategy, which we will refer to as "refurbishment with exchange" or simply "exchange" strategy. The first delivery strategy, i.e. the customer regains his own equipment, is labeled "refurbishment without exchange" or in short "refurbishment" strategy. From a customer point of view, the advantage of refurbishment compared to exchange is the receipt of his own part at the expense of a longer inactive equipment time. From a company point of view, the exchange strategy seems attractive because of lower field technician transportation costs, but this benefit is tempered by higher inventory costs due to the requirement to carry a physical inventory of remanufactured parts. Furthermore, different selling prices can be set for a refurbishment with or without exchange. We allow for a choice and even a mix between the two service delivery strategies. All the decisions with respect to the facility locations, the capacities and the delivery strategy will have an impact on the inventory levels that are needed to obey the contractual obligations. As a consequence, the required inventory levels need to be taken into account when evaluating different design structures. Due to the specific characteristics of remanufacturing, we deal with a one-for-one replenishment inventory problem. Moreover, uncertainty is prevailing in demand, processing and transportation times.

We formulate a non-linear mixed integer model that allows making the decisions concerning the number and type of facilities needed, their capacity levels, the delivery strategy, and the amount of inventory required. Given the model's complexity, we solve it by a differential evolution search algorithm. After presenting the related literature in the next section, Section 4 clarifies the model. There, we consecutively discuss the three sub-problems of the model: the facility location problem, the capacity problem and the inventory problem. Next, in Section 5 the optimization method is briefly reviewed. Section 6 presents the case study that motivates this research. We conclude in Section 7.

\section{Related literature}

One of the major contributions of this paper lies in its multi-disciplinary approach, as we integrate facility location, capacity and inventory decisions. Therefore, our work is related to three distinct research streams: facility location, the queueing and spare part inventory management literature. In this section, we do not want to be exhaustive but review the major contributions in each of the three research fields in order to position our research.

The first relevant literature stream is that of the facility location problem (see for example Melo et al. (2009) for an extensive overview). Many authors have demonstrated that integrating facility location and inventory decisions can be very rewarding (Daskin et al. 2002, Shen et al. 2003). Next to designing a cost efficient supply network, customer service objectives should be taken into consideration, as in the work of Zuo-Jun Max and Daskin (2005), Nozick and 
Turnquist (2001) or Mak and Shen (2009). Although both Nozick and Turnquist (2001) and Mak and Shen (2009) consider stochastic replenishment times, they do not optimize the capacity levels.

The second literature stream deals with queuing networks. Queueing networks are often modeled by using the parametric decomposition approach. The queueing network is decomposed into separate building blocks (i.e. individual workstations or in our case individual facilities in a multi-echelon network). Besides the steady-state waiting time distributions of separate blocks, we have to link the separate blocks by means of linking equations. As such, a linking equation literally links the results obtained at the separate building blocks to obtain the performance of the network as a whole. We refer the reader to Shanthikumar and Buzacott (1981), Buzacott and Shanthikumar (1993), Hopp and Spearman (2000), Whitt (1983) and Whitt (1993) for excellent reviews.

Lastly, the spare part inventory literature has paid special attention to multi-echelon repairable networks due to both the applicability and complexity of these networks. In his seminal work, Sherbrooke (1986) formulates a technique to find the base stock level that minimizes the expected number of back-orders in a two echelon parts network with ample repair capacity. This METRIC model has been adapted by many authors to make it applicable to more realistic settings. Muckstadt (1973) extends the model to the MOD-METRIC that allows multi-indenture parts. The VARIMETRIC model that is based on the work of Graves (1985) and further improved by Sherbrooke (1986), allows for a higher variability in the distribution of the back-orders. A highly restrictive assumption of these multi-echelon models is the conjecture of infinite repair capacity. The research on multi-echelon capacitated repairable networks was started by Gross et al. (1983). Diaz and Fu (1997) optimize the inventory levels for multiple items in a network with limited repair capacity, general repair times and a demand rate that depends on the repair backlog. The work of Sleptchenko et al. (2003) is probably the first that simultaneously optimizes the repair capacity and the inventory levels. Also Caggiano et al. (2006) focus on both capacity and inventory levels in a multi-echelon network, however, they consider the level of capacity and inventory as given and search for an optimal allocation of the resources based on real time data. We deal in our model explicitly with one-for-one replenishment rules. Consequently, (S-1,S) replenishment rules will be applied.

To the best of our knowledge, only Rappold and Van Roo (2009) and van Ommeren et al. (2006) have simultaneously optimized the capacity, the inventory and the facility locations in a multi-echelon repair network with uncertainty. Although van Ommeren et al. (2006) take into account demand uncertainty, they do not consider stochastic processing times and they do not use queuing theory. The setting of our research is comparable to that of Rappold and Van Roo (2009), but it differs on some key aspects. Whereas Rappold and Van Roo (2009) allocate a fixed amount of inventory between the different stocking locations, we determine the required inventory level endogenously based on utilization levels and variances in demand and production processes, while obeying given service levels. Lastly, we formulate a model that is as widely applicable as possible by assuming general distributions for both transportation and repair times and by allowing multiple products, resources and network echelons.

Contrary to earlier work on repair network optimization in which only one delivery strategy is considered, we consider two delivery strategies, i.e. exchange vs. refurbishment. The OEM will incur different costs and will gain different revenues depending on the opted delivery strategy. As such, cost minimization is not a valid objective and consequently we maximize profit. As observed by Melo et al. (2009), a profit maximizing objective is scarcely studied in reverse logistics networks. Recently, increased attention for profit maximization in remanufacturing can be observed, e.g. Vorasayan and Ryan (2006), Jiang et al. (2010), Savaskan et al. (2004), Ferguson and Toktay (2006). However, none of these consider the network design. While Fonseca et al. (2010) do optimize a reverse network design by profit maximization, they focus on waste disposal instead of remanufacturing. Consequently, the profit-orientation of our model can be considered a novelty in the field of network design for remanufacturing activities.

\section{Model Formulation}

In this section, we derive our mathematical model. We first set out the general setting for which the overall model holds (Section 4.1), followed by a description of the flow rates in the network in Section 4.2. These flow rates are commonly used in the three sub-models: the facility location sub-model, the capacity (queueing) sub-model and the 
inventory sub-model. Although we formulate one integrated model that solves these sub-models simultaneously, the three sub-problems will be clarified separately in Sections 4.3 to 4.5 .

\subsection{General Setting}

We consider a stochastic multi-product, single indenture parts network with multiple facility types that can be opened at a location chosen from a fixed, predetermined set of facilities $f(f=1, \ldots, F)$. For this setting, Figure 1 clearly depicts a very general multi-echelon structure. In Section 6 where the case study is introduced, we use a more restricted multi-echelon structure. In this section, however, we prefer to describe the model in its generic form. The figure is an example of a network that consists of three levels, i.e. three types of facilities, but in general the model foresees an unspecified number of levels for each operation to be supported, e.g. cleaning, rewiring, distribution centers, etc.

Customer demand exists for different types of parts, also known as different stock-keeping units (SKUs). The number of SKUs is denoted by $C(c=1, \ldots, C)$. The parts that need to be remanufactured enter the network at customer locations. Moreover, a second type of entry point into the network are the production facilities of new parts. This latter source of parts is necessary to satisfy demand for remanufactured parts when the volume of returned parts is insufficient (see Section 4.2). By combining the part type and the entry point of the part, we can define a part $p$ as an unique combination of a SKU and a SKU-origin. We use $p=1, \ldots, P$ for used parts from customer locations and $p=P+1, \ldots, 2 P$ for newly produced parts from production facilities. As a result, the part set $P$ is a combination of different SKUs (when $C>1$ ) and different SKU-origins. Parts $p$ that belong to a specific SKU $c$ are physically the same part type, but they differ according to the location from which they originate. It is also important to know that a part $p \in\{1, \ldots, P\}$ is exactly the same as a part $p+P$. They only differ in their supply source, i.e. their entry point: a part $p \in\{1, \ldots, P\}$ is supplied by the customer and is remanufactured to an as-new condition, while a part $p+P$ is supplied by the manufacturer and is produced from new components and raw materials.

We assume Poisson distributed demand at the different customer locations, which are represented by echelon level 0 in Figure 1. This assumption is justified when the installed base of complex machines is large compared to the demand levels (Diaz and Fu 1997). With a large installed base, the demand process is not influenced by the lead time of the remanufacturing service.

Remanufacturing activities include several processing steps such as inspection, cleaning, disassembly, rebearing, rewiring, distribution, all performed at one or more facilities $f$. These facilities differ in their level of specialization, i.e. some facilities may only perform one type of operation (e.g. inspection or distribution), while others are all-round facilities and perform multiple operation types. As the level of specialization of the facilities increases, the network will constitute more echelons.

Figure 1: Example of a reverse logistics network.

Obviously, different routings for each part $p$ are possible across the network in Figure 1. A routing is defined by the sequence of facilities (locations) that a part $p$ visits, starting at an entry point and ending in a demand point, 
both located at echelon 0 . If a part is subsequently transported according to the arrows 1,2, 3 and 4, all three echelons (e.g. inspection, rewiring and distribution) are used and the facilities at these echelons can be considered to be specialized. For instance, a facility in echelon $a$ is specialized in inspection. In contrast to this structure, routing the parts through arrows one and five leads to an all-round facility at echelon $a$ that is capable of inspection, remanufacturing and distributing the parts. Consequently, this network structure contains only one echelon. All these possible network structures have in common that the additional inventory of parts needed for an exchange strategy is kept at the highest level in the network, from where the parts are sent directly to the customers. To distinguish between the two service delivery strategies, all routings are duplicated resulting in a set of routings under the refurbishment strategy $\left(r=1, \ldots, R_{p}\right)$, and the exchange strategy $\left(r=R_{p}+1, \ldots, 2 R_{p}\right)$.

Remanufacturing activities performed along a specific routing $r$ for a given part $p$ are represented by $o(o=$ $\left.1, \ldots, O_{p r}\right)$. When multiple process steps are combined into a facility $f$ (e.g. rebearing, rewiring and distribution), it is represented by a single operation $o$. By defining different operations $o$ into a routing $r$, it is possible to construct any general network layout. It becomes even possible to have routings where parts, after being shipped from facility $f$ to another location for some operation, are sent back to the same facility $f$ for subsequent operations. Furthermore, the use of routings $r$ with different numbers of operations, enables us to distinguish network design structures with different echelons, i.e. different levels of specialization in the facilities.

Within these settings, we determine the optimal network structure and the optimal delivery strategy for each service region (customer location), while obeying the main constraints of service level compliance and demand satisfaction. Optimality is defined as profit maximization during a period $T$ in steady-state. Our model formulation relies on three groups of decision variables:

1. binary variables $Y_{f}$ decide on the opening and closing of facilities $f$ (facility location sub-model)

2. integer variables $s_{f}$ decide on the number of operators in each facility $f$ (capacity sub-model)

3. continuous variables $\tau_{p r}$ decide on the flow of parts $p$ that must be assigned to a routing $r$ (overall model)

The first group of decision variables is used in Section 4.3 to open $\left(Y_{f}=1\right)$ or close $\left(Y_{f}=0\right)$ a facility $f$. As remanufacturing activities are generally quite labor intensive, we use labor (operators) as an indicator for the capacity level. Consequently, the second group of decision variables, $s_{f}$, indicates the number of operators at facility $f$ (Section 4.4). Finally, the routing fractions $\tau_{p r}$ indicate which fraction of the incoming flow of parts $p$ will go through the network according to routing $r$. Since the flow rates are commonly used in the three sub-models and $\tau_{p r}$-values influence these flow rates (see Section 4.2), the third group of decision variables handles the optimal flow assigned to the facilities by making a trade-off in the three sub-models between costs for transportation, production, capacity and inventory. The $\tau_{p r}$-values clearly determine the transportation cost between two locations (see Section 4.3), the capacity level of facility $f$ (see Section 4.4) and the inventory level in a routing $r$ (see Section 4.5). Since we use distinct routings for the refurbishment strategy and the exchange strategy, two strategies with different cost and price implications, the decision on $\tau_{p r}$ simultaneously incorporates the decision on the service delivery strategy.

Although our definitions of the decision variables differ from those typically found in comparable literature as described in section 3, these decision variables allow for a model formulation that can be efficiently solved (see section 5). Moreover, our model formulation is very flexible as it allows for different echelons, service delivery strategies and facility types to be considered simultaneously.

\subsection{Flow Rates}

As explained in the previous section, parts can enter the network either from a customer location (used part sent back) or from a production facility (newly manufactured parts). We first discuss the inflow of used parts coming from the customer. Recall that in our setting a technician travels to the customer site, disassembles the used part and sends it to a remanufacturing facility. Due to the high and early involvement of the service provider in the remanufacturing process, the potential return flow equals the demand (not taking into account possible rejections due to quality problems). The 
total demand for refurbished parts at a certain customer location during the period $T$ equals $T * D_{p}$, where $D_{p}$ is the demand rate per time unit for $p \in\{1, \ldots, P\}$.

In order to start the remanufacturing process, some fraction $\tau_{p r}$ of the demand $D_{p}$ has to be assigned to a routing $r$, resulting in an average return rate of part $p$ at such a routing $r$ of

$$
\lambda_{p r}=\tau_{p r} D_{p}
$$

Since the total fraction assigned to all the routings $r$ is not allowed to exceed $100 \%$, we have the following constraint

$$
\sum_{r=1}^{2 R_{p}} \tau_{p r} \leq 1
$$

We note that by summing across all refurbishment routings without exchange ( $\left.r \in\left\{1, \ldots, R_{p}\right\}\right)$ and with exchange $\left(r \in\left\{R_{p}+1, \ldots, 2 R_{p}\right\}\right.$ ), the decision on the fractions $\tau_{p r}$ can result in a mixed policy between the refurbishment strategy and the exchange strategy. Moreover, the inequality in Equation 2 allows for accepting less returns in the network than the maximum volume of $T * D_{p}$, if this would be more economic according to the profit function. Or in other words, the model will decide to provide new parts if it is more profitable than delivering remanufactured ones. For each $p \in\{1, \ldots, P\}$, this leads to a net average arrival rate at the beginning of the reverse chain (external arrivals) of

$$
\lambda_{p}=\sum_{r=1}^{2 R_{p}} \lambda_{p r}
$$

Furthermore, additional units may be disposed of at each subsequent remanufacturing operation because the quality standard is not met. Therefore, a scrap rate $\omega_{\text {pro }}$ is applied to each used part $p \in\{1, \ldots, P\}$ when it leaves operation $o$ in routing $r$, while no scrap rate is assumed for new parts, i.e. $\omega_{\text {pro }}=0 \forall p \in\{P+1, \ldots, 2 P\}$. The average arrival rate of part $p$ at operation $o$ in routing $r$ can then be written has

$$
\begin{array}{ll}
\lambda_{\text {pro }}=\lambda_{\text {pr }} & \text { if } o=1 \\
\lambda_{\text {pro }}=\lambda_{p r} \prod_{\dot{o}=1}^{o-1}\left(1-\omega_{\text {pro }}\right) & \text { if } o>1
\end{array}
$$

Since total demand must be satisfied, new parts $p \in\{P+1, \ldots, 2 P\}$ are necessary to replace scrapped or uncollected parts. The required quantity of new parts is equivalent to the demand level that is not met by remanufactured parts. The delivery rate of remanufactured parts $p \in\{1, \ldots, P\}$ equals $\sum_{r=1}^{2 R_{p}} \lambda_{p r O_{p r}}\left(1-\omega_{p r O_{p r}}\right)$. Consequently, the necessary arrival rate of new parts $p \in\{P+1, \ldots, 2 P\}$ is equal to

$$
\lambda_{p}=D_{(p-P)}-\sum_{r=1}^{2 R_{(p-P)}} \lambda_{(p-P) r O_{(p-P) r}}\left(1-\omega_{(p-P) r O_{(p-P) r}}\right)
$$

Similar to Equation 1, we have to find values $\tau_{p r}$ to determine the flow rate of these new parts arriving from the manufacturing plants. As we want to satisfy total customer demand for remanufactured parts, there are $2 R_{p}-1$ additional decision variables $\tau_{p r}$ for new parts $\left.p \in\{P+1, \ldots, 2 P\}\right)$ under the constraint $\sum_{r=1}^{2 R_{p}-1} \tau_{p r}<1$, where the last routing $2 R_{p}$ takes the remaining units: $\tau_{p\left(2 R_{p}\right)}=1-\sum_{r=1}^{2 R_{p}-1} \tau_{p r}$.

Figure 2 is a graphical, simplified representation of the generic remanufacturing system. Note that the time dimension of planning period $T$ (e.g. days) corresponds to the time dimension of all the flow rates in this section (e.g. parts per day). The remainder of this section is organized as follows. First we develop the three sub-models, one for each problem: the facility location problem, the queueing and capacity problem and the inventory problem. Then these sub-models are integrated into an overall model for profit maximization. 
Figure 2: Conceptual representation of the remanufacturing system.

\subsection{Facility Location Sub-Model}

The first sub-model deals with the number and location of facilities that must be opened according to the model. We use a fixed set $F$ of potential facility locations $f$ and allow for different levels of specialization of these facilities by defining routes with different operations, i.e. sets of activities at these facilities. For example, a facility $f$ at a specific location could be used to execute either inspection or rewiring activities or execute both of these activities. The level of specialization is specified depending on the routing that is chosen according to $\tau_{p r}$. The choice of a location for a facility is a balancing act between different cost components: fixed facility costs, transportation costs and production costs.

\subsubsection{Facility Costs}

The fixed facility costs during planning period $T$ consist of two parts: a fixed overhead cost $F F C 1_{f}$ (e.g. depreciation charges, administration and management) and a fixed cost $F F C 2_{f}$ associated with the number of operators $s_{f}$. If a facility is opened, the fixed costs are charged during each period $T$. The open-close decision is handled by a binary variable $Y_{f}$. Total fixed facility costs $F C$ become

$$
F C=\sum_{f=1}^{F}\left(F F C 1_{f}+F F C 2_{f} s_{f}\right) Y_{f}
$$

The opening or closing of certain facilities has an impact on the routings that can be used in order to fulfill demand. When a facility is closed, no flow is allowed for parts $p$ along a route $r$ that requires facility $f$. As a result, we have

$$
\tau_{p r}=0 \quad \text { if } \exists f: Y_{f}=0 \wedge \sum_{o=1}^{O_{p r}} \varsigma_{p r o f}=1
$$

with $\varsigma_{\text {prof }}$ equal to 1 if operation $o$ for part $p$ in routing $r$ requires facility $f$ and 0 otherwise.

\subsubsection{Transportation Costs}

The transportation costs related to operation $o$ also consist of two parts that are dependent on the facility location: a fixed cost $F T C_{\text {pro }}$ to set-up a shipment and a variable cost $V T C_{\text {pro }}$ that depends on the transportation distance. These costs are charged by the carrier and are the same for refurbished and exchanged parts. They vary with the number of shipments in a planning period $T$. We obtain its total cost value $T C$ as

$$
T C=T \sum_{p=1}^{2 P} \sum_{r=1}^{R_{p}} \sum_{o=1}^{O_{p r}}\left(\lambda_{\text {pro }}+\lambda_{p\left(r+R_{p}\right) o}\right)\left(F T C_{\text {pro }}+V T C_{\text {pro }}\right)
$$




\subsubsection{Production Costs}

The decision where to open facilities and which activities to allocate to these facilities will also impact the production costs. For each unit of part $p$ that is processed through operation $o$, a variable production cost for resources consumed $V P C_{\text {pro }}$ has to be included. Moreover, as explained in Section 4.2, remanufacturing operations are characterized by some fractional scrap level $\omega_{\text {pro }}$ to handle the quality problems. For each scrapped unit, a variable disposal cost $V D C_{\text {pro }}$ is added to the total production cost $P C$. Consequently, $P C$ can be written as

$$
P C=T \sum_{p=1}^{2 P} \sum_{r=1}^{2 R_{p}} \sum_{o=1}^{O_{p r}} \lambda_{\text {pro }}\left(V P C_{\text {pro }}+\omega_{\text {pro }} V D C_{\text {pro }}\right)
$$

\subsection{Queueing and Capacity Sub-Model}

The model wants to determine an appropriate combination of capacity and inventory investments. To this end, we need to describe the relationship between capacity levels and lead times at the facilities in the network. We do this by transforming the network into a queuing system that consists of arrival and remanufacturing processes.

\subsubsection{Arrival Processes}

From Section 4.2, we know that the system is characterized by an expected arrival rate $\lambda_{p}$ for each part $p$. This is equivalent to an expected time between arrivals of $I A_{p}=1 / \lambda_{p}$. Given a large installed base of complex machines, it is reasonable to assume that the arrivals follow a Poisson process with parameter $\lambda_{p}$. This Markovian arrival pattern leads to a squared coefficient of variation (SCV) at the entry points of $c_{I A_{p}}^{2}=1$, a measure that describes the variability level of the interarrival times. As a result, assigning fractions $\tau_{p r}$ to routes $r$ does not change the SCV of the interarrival times at the entry point of these individual routes, referred to by $c_{I A_{p r}}^{2}$ (Whitt 1983). Such an entry point can then be modeled as an M/G/m queue. However, higher echelon facilities will face a variability at their inbound that depends on the variability and utilization levels at all lower echelon facilities (see Equation 10). Since the arrival process at these facilities is no longer Poisson, the resulting queues are modeled as $\mathrm{G} / \mathrm{G} / \mathrm{m}$ queues.

The next step is to aggregate at each facility $f$, the rates of all arriving parts $p$ into a single arrival process. This results in an average aggregate arrival rate $\lambda_{f}$ and a SCV of the aggregate interarrival times $c_{I A_{f}}^{2}$ at a facility $f$. When we define the aggregate arrival rate of part $p$ at facility $f$ as $\lambda_{p f}=\sum_{r=1}^{2 R_{p} O_{p r}} \sum_{o=1} \lambda_{p r o} \varsigma_{p r o f}$, we have $\lambda_{f}=\sum_{p=1}^{2 P} \lambda_{p f}$. It consists of facility arrivals that are both internal (i.e. coming from another facility) and external (i.e. coming from the customer). The external aggregate arrival rate at facility $f$ is determined by $\lambda_{f}^{\prime}=\sum_{p=1}^{2 P} \sum_{r=1}^{2 R_{p}} \lambda_{p r 1} \varsigma_{p r 1 f}$. The SCV of the external aggregate interarrival times $c_{I A_{f}}^{\prime 2}$ is also equal to 1 due to the Markovian arrival process, but the value of the internal aggregate interarrival times $c_{I A_{f}}^{2}$ depends on the variability and utilization levels of production processes along a part's route. Expressions for these values are derived in the next section.

\subsubsection{Remanufacturing Processes}

In this section we elaborate on the relationship between capacity levels and lead times of the operations. For each part $p$ that requires an operation $o$ along route $r$, we have the following production characteristics: expected unit processing time $P R_{\text {pro }}$ with $\mathrm{SCV} c_{P R_{p r o}}^{2}$ and variance $\sigma_{P R_{\text {pro }}}^{2}$. The expected unit process rate $\mu_{\text {pro }}$ equals $1 / P R_{\text {pro }}$. As we work with single unit flows, possible set-up times that occur when processing different parts, can be incorporated into the processing time and the corresponding SCV and variance. We allow the processing time characteristics to be part dependent: not only different SKUs $c$ differ in remanufacturing process times due to e.g. part size or complexity, 
also the SKU-origin has an impact on the required remanufacturing times. Depending on their origin, parts may have been exposed to different working conditions such as heat and moisture and may have received different preventive maintenance treatments.

In our stochastic model, we have to take into account possible disruptions resulting in a reduced availability of capacity. Therefore, we transform the natural expected value and variability of the processing time into effective measures using the capacity availability $A_{f}$ (Hopp and Spearman 2000). We have

$$
\begin{aligned}
A_{f} & =\frac{\left(W_{f}-P M_{f}\right)\left(M T B F_{f} /\left(M T T R_{f}+M T B F_{f}\right)\right) E_{f}}{T} \\
P R e_{\text {pro }} & =P R_{\text {pro }} /\left(\sum_{f} A_{f} \varsigma_{\text {prof }}\right) \\
\sigma_{\text {PRe pro }}^{2} & =c_{P R_{\text {pro }}}^{2} P \operatorname{Re}_{\text {pro }}^{2}+2 P \operatorname{Re}_{\text {pro }} \sum_{f} M T T R_{f}\left(1-A_{f}\right) \varsigma_{\text {prof }}
\end{aligned}
$$

The availability of capacity depends on the characteristics of the required machinery at facility $f$, i.e. the MeanTime-To-Repair $\left(M T T R_{f}\right)$, the Mean-Time-Between-Failures $\left(M T B F_{f}\right)$, the number of working time units $\left(W_{f}\right)$, the number of time units for preventive maintenance $\left(P M_{f}\right)$ and the factor $E_{f}$ to deal with the residual efficiency losses. Likewise, one could state more generally that availability depends on the number of potential working time units $\left(W_{f}\right)$ minus the planned interruptions e.g. labor union meetings, multiplied by a correcting factor for unplanned interruptions $\left(M T B F_{f} /\left(M T T R_{f}+M T B F_{f}\right)\right)$ and residual inefficiencies $\left(E_{f}\right)$. Note that all these characteristics for route $r$ are exactly the same as for route $r+R_{p}$ because there is no difference in the remanufacturing process for a refurbishment and an exchange strategy.

As with the arrival processes, we need to aggregate the processing characteristics of all the parts $p$ at a facility level. The expression for the average process time at facility $f$ is a weighted average over all the parts across all the routings and all the operations performed in facility $f$

$$
P R_{f}=1 / \mu_{f}=\sum_{p=1}^{2 P} \sum_{r=1}^{R_{p}} \sum_{o=1}^{O_{p r}} \frac{\left(\lambda_{\text {pro }} \varsigma_{\text {prof }}+\lambda_{p\left(r+R_{p}\right) o} \varsigma_{p\left(r+R_{p}\right) o f}\right)}{\lambda_{f}} P \operatorname{Re}_{\text {pro }}
$$

while its SCV becomes

$$
\begin{aligned}
c_{f}^{2}= & {\left[\sum_{p=1}^{2 P} \sum_{r=1}^{R_{p}} \sum_{o=1}^{O_{p r}} \frac{\left(\lambda_{\text {pro }} \varsigma_{\text {prof }}+\lambda_{\left.p\left(r+R_{p}\right) o \varsigma_{p\left(r+R_{p}\right) o f}\right)}\right.}{\lambda_{f}}\left(P R e_{\text {pro }}\right)^{2}\right] \mu_{f}^{2}-1+} \\
& \sum_{p=1}^{2 P} \sum_{r=1}^{R_{p}} \sum_{o=1}^{O_{p r}} \frac{\left(\lambda_{p r o} \varsigma_{p r o f}+\lambda_{\left.p\left(r+R_{p}\right) o \varsigma_{p\left(r+R_{p}\right) o f}\right)}\right.}{\lambda_{f}} \frac{\sigma_{P R e_{p r o}}^{2}}{\left(P R e_{p r o}\right)^{2}}
\end{aligned}
$$

Apart from flow rates and processing times, the capacity level $s_{f}$ and the resulting utilization level $\rho_{f}$ at a facility $f$ will also impact its lead time of remanufacturing parts. The facility utilization is determined by the following equation, also known as the adapted traffic intensity

$$
\begin{aligned}
& \rho_{f}=\frac{\lambda_{f}}{\mu_{f} s_{f}} \\
& =\sum_{p=1}^{2 P} \sum_{r=1}^{R_{p}} \sum_{o=1}^{O_{p r}}\left(\lambda_{\text {pro }} \varsigma_{\text {prof }}+\lambda_{p\left(r+R_{p}\right) o \varsigma_{p}\left(r+R_{p}\right) o f}\right) P R e_{\text {pro }} / s_{f} \leq 1
\end{aligned}
$$

The final input parameter for the queueing network model is the SCV of the aggregate interarrival times at facility $f$ or $c_{I A_{f}}^{2}$. This can be derived if we know the variability of interdeparture times of parts leaving the lower echelon facility $f^{\prime}$. Buzacott and Shanthikumar (1993) have presented several approximations for systems with multiple servers, but we opt for the following linking equation (Hopp and Spearman 2000):

$$
c_{I D_{f^{\prime}}}^{2} \approx 1+\left(1-\rho_{f^{\prime}}^{2}\right)\left(c_{I A_{f^{\prime}}}^{2}-1\right)+\frac{\rho_{f^{\prime}}^{2}}{\sqrt{s_{f^{\prime}}}}\left(c_{f^{\prime}}^{2}-1\right)
$$


which not only depends on the facility utilization, but also on its production and arrival process variability level.

Next, we use a result from Shanthikumar and Buzacott (1981) for the relationship between $c_{I D_{f^{\prime}}}^{2}$ and the SCV of the aggregate interarrival time at facility $f$ for units coming from facility $f^{\prime}$, represented by $c_{I A_{f^{\prime} f}}^{2}$. This depends on $\pi_{f^{\prime} f}$, the fraction that is going from facility $f^{\prime}$ to $f$

$$
c_{I A_{f^{\prime} f}}^{2}=\pi_{f^{\prime} f} c_{I D_{f^{\prime}}}^{2}+\left(1-\pi_{f^{\prime} f}\right)
$$

The expression for $c_{I A_{f}}^{2}$ is the sum of a weighted average of $c_{I A_{f}}^{\prime 2}$ and $c_{I A_{f^{\prime} f}}^{2}$

$$
c_{I A_{f}}^{2}=\sum_{f^{\prime}}\left(\frac{\lambda_{f^{\prime}}}{\lambda_{f}} \pi_{f^{\prime} f}\right) c_{I A_{f^{\prime} f}}^{2}+\frac{\lambda_{f}^{\prime}}{\lambda_{f}} c_{I A_{f}}^{\prime 2}
$$

which, based on Equation 11 with $c_{I A_{f^{\prime} f}}^{2}$ replaced by 1 (external Poisson arrivals), becomes

$$
=\sum_{f^{\prime}}\left(\frac{\lambda_{f^{\prime}}}{\lambda_{f}} \pi_{f^{\prime} f}\right)\left(\pi_{f^{\prime} f} c_{I D_{f^{\prime}}}^{2}+\left(1-\pi_{f^{\prime} f}\right)\right)+\frac{\lambda_{f}^{\prime}}{\lambda_{f}}
$$

and when combined with Equation 10 results in

$$
\approx \sum_{f^{\prime}}\left(\frac{\lambda_{f^{\prime}}}{\lambda_{f}} \pi_{f^{\prime} f}\right)\left(\pi_{f^{\prime} f}\left[1+\left(1-\rho_{f^{\prime}}^{2}\right)\left(c_{I A_{f^{\prime}}}^{2}-1\right)+\frac{\rho_{f^{\prime}}^{2}}{\sqrt{s_{f^{\prime}}}}\left(c_{f^{\prime}}^{2}-1\right)\right]+\left(1-\pi_{f^{\prime} f}\right)\right)+\frac{\lambda_{f}^{\prime}}{\lambda_{f}}
$$

Reorganization of Equation 12 when expressed as an equality, leads to $F$ linear equations with $F$ unknown parameters $c_{I A_{f}}^{2}$

$$
\forall f: \quad-\sum_{f^{\prime}} \lambda_{f^{\prime}} \pi_{f^{\prime} f}^{2}\left(1-\rho_{f^{\prime}}^{2}\right) c_{I A_{f^{\prime}}}^{2}+\lambda_{f} c_{I A_{f}}^{2}=\sum_{f^{\prime}} \lambda_{f^{\prime}} \pi_{f^{\prime} f}\left(\pi_{f^{\prime} f} \rho_{f^{\prime}}^{2}\left(1+\frac{c_{f^{\prime}}^{2}-1}{\sqrt{s_{f^{\prime}}}}\right)+1-\pi_{f^{\prime} f}\right)+\lambda_{f}^{\prime}
$$

To solve these equations and to find values for $c_{I A_{f}}^{2}$, we have to obtain the transition matrix, which consists of 1. the proportion of units from outside the system (stage 0 ) and going to the first facility in the routing (stage $f$ )

$$
\pi_{0 f}=\lambda_{f}^{\prime} / \sum_{f} \lambda_{f}^{\prime}
$$

2. the proportion of units from facility $f^{\prime}$ (stage $f^{\prime}$ ) and going to the next facility in the routing (stage $f$ )

$$
\pi_{f^{\prime} f}=\sum_{p=1}^{2 P} \sum_{r=1}^{2 R_{p}} \sum_{o=1}^{O_{p r}-1} \lambda_{p r o}\left(1-\omega_{p r o}\right) \varsigma_{p r o f^{\prime}} \varsigma_{p r(o+1) f} / \lambda_{f^{\prime}}
$$

3. the proportion of units from the last facility in the routing (stage $f$ ) and leaving the system (stage 0 )

$$
\pi_{f^{\prime} 0}=\sum_{p=1}^{2 P} \sum_{r=1}^{2 R_{p}} \lambda_{p r O_{p r}}\left(1-\omega_{p r O_{p r}}\right) \varsigma_{p r O_{p r} f^{\prime}} / \lambda_{f^{\prime}}
$$

At this point we are able to estimate the expected lead time for part $p$ at an operation $o$ along route $r$, which takes place at facility $f$, by using the approximation from Whitt (1993). It is an accurate GI/G/m-model with a correction factor $\phi$ that applies to a system under heavy traffic conditions with multiple parallel servers (Hopp and Spearman 2000). We refer to the Appendix A for more details about this factor $\phi$. Equation 14 provides us with the relationship between the expected lead time of an operation in function of the capacity decision. 


$$
E W_{\text {pro }} \approx \sum_{f} E W Q_{f} \varsigma_{\text {prof }}+P R e_{\text {pro }}
$$

with

$$
E W Q_{f} \approx \phi\left(\rho_{f}, c_{I A_{f}}^{2}, c_{f}^{2}, s_{f}\right)\left(\frac{c_{I A_{f}}^{2}+c_{f}^{2}}{2}\right)\left(\frac{\rho_{f}^{\sqrt{2\left(s_{f}+1\right)}-1}}{s_{f}\left(1-\rho_{f}\right)}\right) P R_{f}
$$

Since the lead time clearly depends on capacity, the capacity decision can be linked with customer service and inventory levels.

\subsection{Inventory Sub-Model}

At this point, the relationships between the facility location and the corresponding costs on the one hand and the capacity decisions and lead times on the other hand have been explained. Now, appropriate inventory levels have to be set in order to meet the service levels. We first determine the inventory level for the refurbishment strategy with an exchange option. Next, we adapt the reasoning to provide the inventory level for the refurbishment strategy without an exchange option.

When executing an exchange strategy, an inventory of parts that can be shipped directly to the customers is required. Contrary to many settings described in the literature, our model deals with a network where this inventory is held at the highest echelon of a selected route. This characteristic is motivated by the case-study. Consequently, all inventory, except the inventory in process, resides at the highest echelon in a route. Each part ordered by a customer will be sent from the highest echelon directly to the customer location. The shipment of the part is coordinated with the arrival of a field technician at the customer site. Next, the technician disassembles the machine and replaces the used part. The removed part is sent to a remanufacturing center to be processed and to replenish the inventory of as-new parts. Therefore, there is an automatic replenishment of the inventory by the take-back of the used part. Consequently, the inventory model can be considered to be a continuous review, one-for-one replenishment policy, i.e. an $(S-1, S)$ inventory system, in which $S$ is the order-up-to level. Note that in case the used part is scrapped, the inventory is replenished by adding a newly produced part. Moreover, the assumption of a transportation quantity of one unit further supports this type of inventory system. Figure 3 gives a graphical representation of the inventory residing in the remanufacturing network. In essence three types of inventory can be identified in case of refurbishment with exchange services (left hand side of Figure 3). First, some parts will be in transport or being remanufactured. Secondly, due to limited capacity at the facilities some parts will be waiting at a facility until processing capacity becomes available. Lastly, as exchange service includes the instantaneous exchange of the used part by an already remanufactured part, a sufficient number of as-new parts must be available at the highest echelon for direct shipment to customer locations (this inventory is indicated as "commercial inventory").

Figure 3: Representation of inventory across remanufacturing network. 
Similar to Nozick and Turnquist (2001)), we derive appropriate order-up-to levels $S$ based on a target stock-out probability. The order-up-to level $S$ exists of two components: inventory to cover the expected demand during total lead time, further referred to as $\hat{\mu}_{D D L T}$, and additional inventory at the end of each routing $r \in\left\{R_{p}+1, \ldots, 2 R_{p}\right\}$ to guarantee a specific service level, further referred to as safety stock $(\hat{S S})$. So we have $S=\hat{\mu}_{D D L T}+\hat{S S}$, and to estimate values for $\hat{\mu}_{D D L T}$ and $\hat{S S}$, several steps are required.

The first step is determining the total expected lead time along a route $r$ for each part $p$ as

$$
L T_{p r} \approx \sum_{o=1}^{O_{p r}}\left(T R_{p r o}+E W_{p r o}\right)
$$

and its variance as

$$
\sigma_{L T_{p r}}^{2} \approx \sum_{o=1}^{O_{p r}}\left(\sigma_{T R_{p r o}}^{2}+\sum_{f} \sigma_{W Q_{f}}^{2} \varsigma_{p r o f}+\sigma_{P R_{p r o}}^{2}\right)
$$

where

$$
\sigma_{T R_{\text {pro }}}^{2}=c_{T R_{\text {pro }}}^{2} T R_{\text {pro }}^{2}
$$

and where the transportation process related to each operation $o$ is characterized by an expected transportation time $T R_{p r o}$ and an associated SCV $c_{T R_{p r o}}^{2}$. From these two parameters, its variance $\sigma_{T R_{p r o}}^{2}$ can be derived. For the variance of the facility waiting time $\sigma_{W Q_{f}}^{2}$, we opt for the approximation from Whitt (1993) (see Appendix B).

We recall that we have defined a part $p$ as a unique combination of a SKU $c$ and an entry point. Clearly, inventory is held for each of the SKUs separately, but irrespectively of the entry point (demand origin). For example, if demand for a specific part type exists in two regions which are both supplied by the same facility, both regions will obtain parts from the same inventory at that facility. Therefore, the next step is to obtain an aggregated total expected lead time of a SKU $c$. This is based on the total expected lead time of all parts $p$ that belong to the SKU $c$, indicated by a parameter $v_{c p}=1$. When part $p$ does not belong to SKU $c$, we have $v_{c p}=0$. The lead time experienced by a SKU $c$ when it reaches the outbound of the highest echelon facility $f$ (i.e. at operation $O_{p r}$ ) equals

$$
L T_{c f} \approx \sum_{p=1}^{2 P} v_{c p} \sum_{r=R_{p}+1}^{2 R_{p}} \frac{\lambda_{p r O_{p r}}\left(1-\omega_{p r O_{p r}}\right) \varsigma_{p r O_{p r} f}}{\lambda_{c f}} L T_{p r}
$$

and its variance is

$$
\sigma_{L T_{c f}}^{2} \approx \sum_{p=1}^{2 P} v_{c p} \sum_{r=R_{p}+1}^{2 R_{p}} \frac{\lambda_{p r O_{p r}}\left(1-\omega_{p r O_{p r}}\right) \varsigma_{p r O_{p r} f}}{\lambda_{c f}}\left[L T_{p r}^{2}+\sigma_{L T_{p r}}^{2}\left(\frac{L T_{c f}^{2}}{L T_{p r}}\right)^{2}\right]-L T_{c f}^{2}
$$

where

$$
\lambda_{c f}=\sum_{p=1}^{2 P} v_{c p} \sum_{r=R_{p}+1}^{2 R_{p}} \lambda_{p r O_{p r}}\left(1-\omega_{p r O_{p r}}\right) \varsigma_{p r O_{p r} f}
$$

We refer to Equation 8 to explain the derivation of this aggregate variance, knowing that $c_{c f}^{2}=\sigma_{L T_{c f}}^{2} / L T_{c f}^{2}$.

Once the lead times are known, the final step is to determine the order-up-to level $S_{c f}$ that must be available to satisfy, with a service level of $x_{c f} \%$, the demand for parts of SKU $c$, where facility $f$ is used at the end of an 'exchange'routing. To this end, we need the distribution of the demand during the lead time. Nozick and Turnquist (2001) use Palm's Theorem to justify their choice for a Poisson distributed demand during lead time. However, Palm's Theorem relies on the assumption of ample (infinite) capacity. As we take capacity decisions explicitly into account, the use of a Poisson distributed demand during lead time is in disagreement with the queueing relationships in our model. The distribution of the demand during lead time is a convolution of the demand distribution, which we assume to be Poisson, and the lead time distribution, which we assume to be lognormal. These assumptions are confirmed by Diaz 
and Fu (1997) for the Poisson arrivals and Lambrecht et al. (1998) for the lognormality of the lead times. In order to identify a parametric distribution for the demand during lead time, we follow the approach of Tadikamalla (1984). When the demand distribution and the lead time distribution are not known, they propose the lognormal, the gamma and the Weibull as adequate distributions for the demand during lead time. When both distributions are known, which is the case here (Poisson and lognormal respectively), they suggest a mathematical expression followed by the selection of the best approximate distribution. Since a closed form for this mathematical expression is not guaranteed, we have conducted a simulation study to find the general, best fit under our distribution assumptions. This approach is also viable because of its computational simplicity. First, observations are sampled from both a Poisson and a lognormal distribution with different parameters, i.e. Poisson arrivals with parameters $\{0.25,0.5,0.75,1,2,4,6,8\}$ for the daily rates in combination with a lognormal lead time with a fixed mean of 10 days and standard deviation levels of $\{1,5,10,15\}$ (in total 32 scenarios are considered). Next, the observations are convoluted to calculate the demand during the lead time and a set of parametric distributions is fitted to the demand data. Figure 4 summarizes the results for different distributions. Conform with the results of Tadikamalla (1984), we observe that the Weibull distribution performs strongly, however, the Beta distribution has a even better and more stable performance. Consequently, we opt to use the Beta distribution to approximate the distribution of the demand during lead time.

Figure 4: Summary of the experimental results for the demand during lead time distribution.

Two parameters $\alpha_{c f}$ and $\beta_{c f}$ describe the mean $\hat{\mu}_{D D L T_{c f}}$ and the variance $\hat{\sigma}_{D D L T_{c f}}^{2}$ of the Beta distribution for the demand during the lead time according to

$$
\begin{aligned}
\hat{\mu}_{D D L T_{c f}} & =\frac{\alpha_{c f}}{\alpha_{c f}+\beta_{c f}} \\
\hat{\sigma}_{D D L T_{c f}}^{2} & =\frac{\alpha_{c f} \beta_{c f}}{\left(\alpha_{c f}+\beta_{c f}\right)^{2}\left(\alpha_{c f}+\beta_{c f}+1\right)}
\end{aligned}
$$

with $\hat{\mu}_{D D L T_{c f}} \approx \lambda_{c f} L T_{c f}$ (Little's law) and $\hat{\sigma}_{D D L T_{c f}}^{2} \approx \sigma_{D_{c f}}^{2} L T_{c f}+\sigma_{L T_{c f}}^{2} \lambda_{c f}^{2}$, and where $\sigma_{D_{c f}}^{2}$ is the variance of the demand for SKU $c$ with facility $f$ at the highest echelon. For the Poisson distributed demand, we have $\sigma_{D_{c f}}^{2}=\lambda_{c f}$. Solving both equations for $\alpha_{c f}$ and $\beta_{c f}$ leads to

$$
\begin{aligned}
& \alpha_{c f}=\hat{\mu}_{D D L T_{c f}}\left(\frac{\hat{\mu}_{D D L T_{c f}}\left(1-\hat{\mu}_{D D L T_{c f}}\right)}{\hat{\sigma}_{D D L T_{c f}}^{2}}-1\right) \\
& \beta_{c f}=\left(1-\hat{\mu}_{D D L T_{c f}}\right)\left(\frac{\hat{\mu}_{D D L T_{c f}}\left(1-\hat{\mu}_{D D L T_{c f}}\right)}{\hat{\sigma}_{D D L T_{c f}}^{2}}-1\right)
\end{aligned}
$$


The $x_{c f} \%$ quantile of the beta distribution $q b e t a\left(x_{c f} \%, \alpha_{c f}, \beta_{c f}\right)$ determines the level of $S_{c f}$. Note that $S_{c f}$ is also equal to $\hat{\mu}_{D D L T_{c f}}+\hat{S S} S_{c f}$, so we have $\hat{S S} S_{c f}=q \operatorname{beta}\left(x_{c f} \%, \alpha_{c f}, \beta_{c f}\right)-\hat{\mu}_{D D L T_{c f}}$.

This $S$-level ensures that the output rate at the end of the system can be sustained with a service level of $x_{c f} \%$. It can be split into two components: $\hat{\mu}_{D D L T_{c f}}$, which naturally arises at any facility $f$ along a route $r \in\left\{R_{p}+1, \ldots, 2 R_{p}\right\}$, and $S \hat{S}_{c f}$, which is held at the end of the system. Nevertheless, when there is scrap in a remanufacturing route, the flow rates along this route are larger than its output rate. As a result, more inventory arises naturally along such a route. If we define $E N_{\text {pro }}$ as the total expected number of parts $p$ at each operation $o$ in these routings $r$, we can determine their value by applying their total expected arrival rates to the total expected delays (Little's Law). These delays are the transportation time $T R_{\text {pro }}$ and the lead time from Equation 14. We have

$$
E N_{\text {pro }} \approx \lambda_{\text {pro }}\left(T R_{\text {pro }}+E W_{\text {pro }}\right)
$$

Finally, the total inventory costs $I C 1$ in the refurbishment network with exchange becomes

$$
I C 1 \approx \sum_{p=1}^{2 P} \sum_{r=R_{p}+1}^{2 R_{p}} \sum_{o=1}^{O_{p r}} H C_{\text {pro }} E N_{\text {pro }}+\sum_{c=1}^{C} \sum_{f=1}^{F} H C_{c f} \hat{S S_{c f}}
$$

where $H C_{p r o}$ and $H C_{c f}$ are defined as the cost to keep one unit in stock during planning period $T . H C_{c f}$ should be interpreted as an aggregate unit inventory cost obtained by Equation 16 with $L T_{p r}$ replaced by $H C_{p r} O_{p r}$. Note that all $E N_{\text {pro }}$ units are part of $\hat{\mu}_{D D L T_{c f}}$ when there is no scrap from this operation onwards in route $r$, while only a fraction of $E N_{\text {pro }}$ is part of $\hat{\mu}_{D D L T_{c f}}$ when that is the case.

In contrast to the refurbishment with exchange, the refurbishment without exchange does not require the service provider to maintain an inventory of remanufactured parts (see also right hand side of Figure 3). Consequently, the inventory in that network type is limited to work in process, which is equal to $E N_{\text {pro }}$. The inventory costs can then be written as

$$
I C 2 \approx \sum_{p=1}^{2 P} \sum_{r=1}^{R_{p}} \sum_{o=1}^{O_{p r}} H C_{\text {pro }} E N_{\text {pro }}
$$

with $E N_{\text {pro }}$ similar to Equation 17, but for routings $r \in\left\{1, \ldots, R_{p}\right\}$.

Total inventory cost equals $I C \approx I C 1+I C 2$.

\subsection{Profit Maximization Model}

All the relationships discussed so far can be combined to characterize all trade-offs involved. As we want to take a profit perspective, we still need to determine the expected revenue. It clearly depends on the extent to which the refurbishment demand is fulfilled by a delivery strategy with or without an exchange option. We have

$$
R E V=T \sum_{p=1}^{2 P} \sum_{r=1}^{2 R_{p}}\left(1-\omega_{p r O_{p r}}\right) \lambda_{p r O_{p r}} S P_{p r}
$$

Revenue from part $p(p=\{1, \ldots, 2 P\})$ is generated in a routing $r\left(r=\left\{1, \ldots, 2 R_{p}\right\}\right)$ by multiplying the part specific unit selling price $S P_{p r}$ by its volume sold. This volume equals the departure rate at the last operation in route $r\left(\left(1-\omega_{p r O_{p r}}\right) \lambda_{p r O_{p r}}\right)$ times the number of time units $T$. We know from Section 4.2 that the total volume delivered to the market must match its total demand $T * D_{p}$. As a results, also new parts $p \in\{P+1, \ldots, 2 P\}$ are delivered to satisfy the demand that is not covered by remanufactured parts. The selling price $S P_{p r}$ can differ between parts and even between routings. This is useful to distinguish between regional prices and to charge a price depending on the service delivery strategy. We can argue that a customer's willingness to pay is higher for refurbishment with an exchange because of the faster response time (delivery from stock). For a refurbishment service without an exchanged 
part, the customer has to wait until the entire remanufacturing process is finished, leading to a prolonged machine down time. However, a customer's request to receive its own, initial part may result in a higher willingness to pay for refurbishment without exchange.

In this work, we maximize profit for the remanufacturing business without taking into consideration the new equipment business. Although in some settings it can be argued that increased remanufacturing services will negatively impact new equipment sales, we do not consider this potential cannibalization of new sales. This assumption is justified in our case study due to the intense competition in the remanufacturing business, i.e. if the company does not remanufacture the part, the company's competitors will perform the service.

Finally, another cost component of the objective function must still be established to cover cost differences between refurbishment strategies with and without the exchange option. On the one hand, the exchange strategy is characterized by higher inventory costs (Section 4.5), but on the other hand, it is characterized by a field technician process time that is only half the time needed for a refurbishment without exchange. Whereas for an exchange the technician visits the customer only once, two interventions are required for a refurbishment without exchange: one intervention to retrieve the part and another intervention to re-install the part. Since the difference in inventory costs is already taken into account, we formulate the transportation and labor costs of the technicians $(\mathrm{TeC})$ here

$$
T e C=T \sum_{p=1}^{2 P} \sum_{r=1}^{2 R_{p}}\left(1-\omega_{p r O_{p r}}\right) \lambda_{p r O_{p r}} F T e C_{p r O_{p r}}
$$

Since total volume to be uninstalled equals total volume to be installed, we focus on the net volume that exits the last operation in each routing of part $p$. We distinguish between the different number of required field trips in both refurbishment strategies by defining a fixed cost $F T e C_{p r O} O_{p r}$ for a strategy without exchange that is twice the value of $F T e C_{p r O_{p r}}$ for a strategy with exchange.Equations 3, 5,6, 18, 19, 20 and 21 can be summarized in the profit objective function

$$
\text { Profit } \approx R E V-F C-T C-P C-I C-T e C
$$

We maximize the profit subject to equations $2,4,9$ and the constraints $0 \leq \tau_{p r} \leq 1 \forall \tau_{p r}$.

\section{Model Optimization}

The network optimization model that is described in Section 4 consists of three difficult sub-problems that are simultaneously solved, subject to the goal of overall network profit maximization:

1. the facility location problem: binary variables $Y_{f}$ decide on the opening and closing of facilities

2. the capacity problem: integer variables $s_{f}$ decide on the number of servers in each facility

3. the inventory problem: the $(S-1, S)$ parameters decide on the inventory position

The continuous variables $\tau_{p r}$ that decide on the flow of each part $p$ to be assigned to each routing $r$, drive the overall model towards a solution that is optimal from the perspective of the three sub-problems simultaneously. At the same time an optimal mix is obtained of new and as-new parts as well as an optimal mix of refurbishment service policies with and without exchange.

Moreover, we have a non-linear objective function and non-linear constraints. The queueing formulas for the expected waiting times are not only non-linearly dependent on the utilization level $\rho_{f}$, which depends on the decision variables $s_{f}$ and $\tau_{p r}$, the numerous conditional relationships in the correction factor $\phi\left(\rho_{f}, c_{I A_{f}}^{2}, c_{f}^{2}, s_{f}\right)$ make it difficult to solve. As a result, the model can be classified as a mixed integer non-linear programming problem (MINLP). Finding a 
global optimum for such problems is hard because all the difficulties of two NP-complete sub-problems are combined: the combinatorial nature of discrete programs and the difficulty in solving non-linear programs. The computational complexity not only grows exponentially with the number of discrete variables and the number of decisions within each discrete variable, but also with the number of variables creating non-linearities into the model.

The methods for solving MINLP problems are usually divided into two main categories: deterministic and stochastic solution methods. All these methods use a structured search process, but they differ in the way they run through it. Deterministic methods, such as branch-and-reduce (Ryoo and Sahinidis 1995) and (Ryoo and Sahinidis 1996), the $\alpha \mathrm{BB}$ branch-and-bound (Adjiman et al. 1997) and (Androulakis et al. 1995), Barton's branch-and-cut (Kesavan and Barton 2000), interval analysis based methods (Vaidyanathan and El-Halwagi 1996) and the extended cutting plane (Westerlund et al. 1998), run through the algorithm in a predefined way, which means that for a given set of algorithm control parameters, the next step of computation is always exactly known and determined. On the other hand, stochastic global optimization methods (also known as adaptive random search methods), such as Differential Evolution (Storn and Price 1997), adaptive Lagrange-multiplier methods (Wah and Wang 1999), simulated and nested annealing (Rajasekaran 2000), ant colony simulation (Mathur et al. 2000) and tabu search (Chelouah and Siarry 2000), run through the algorithm in a way that is unknown in advance, which means that for a given set of algorithm control parameters, the next step of computation is always unknown and undetermined.

After studying seven difficult design and control MINLP problems in chemical engineering, Babu and Angira (2002) conclude that the technique of Differential Evolution (DE) heuristic, which is a member of the broader family of genetic algorithms, is the best evolutionary computation method. According to Lampinen and Zelinka (1999), DE outperforms any of the competing methods like branch \& bound using sequential quadratic programming, integerdiscrete-continuous non-linear programming, simulated annealing, genetic algorithm, non-linear mixed-discrete programming, etc., also for hard non-linear objective functions with multiple non-trivial constraints.

Lieckens and Vandaele (2007) have shown that DE also performs very well for a stochastic reverse logistics network design model. As a result, we opt to use it as an appropriate solution method for our network design problem. This choice is also motivated by the following DE characteristics (Storn and Price 1997)

- it is simple, fast and robust;

- it has a superior global optimization ability;

- it can easily be implemented in a parallel computing environment to speed up the optimization;

- it is effective in non-linear optimization and can be very easily adapted for mixed parameter optimization;

- it handles undifferentiable objective functions;

- it operates on flat surfaces;

- it can provide multiple solutions in a single run.

Briefly summarized, the first step of DE is the creation of an initial population of different elements. Each element contains a value for each decision variable $\left(Y_{f}, s_{f}\right.$ and $\left.\tau_{p r}\right)$, randomly selected between relevant lower and upper bounds. Feasible solutions are initially not guaranteed, but it is the difference of randomly sampled population elements in combination with a constraint handling procedure that drives the mutation and recombination process towards better performing childs in the population. Different mutation schemes can be selected, but they all reflect information of the objective function that is being optimized. Instead of using only local information for each population element, DE mutates all elements with the same universal distribution. In this way, the whole search space is covered and a global optimum can be found. Another advantage of DE for mixed integer problems is that discrete variables $\left(Y_{f}\right.$ and $\left.s_{f}\right)$ are treated as continuous values to create subsequent childs (this maintains the diversity of the population), while they are rounded at the moment of objective function evaluation (this avoids sub-optimal results because only feasible solutions give feedback to the optimization process). We refer to Lieckens and Vandaele (2007) for more details about how the algorithm is applied. 


\section{Case study}

Our research is motivated by the challenge of designing a remanufacturing network at a worldwide manufacturer of construction, mining and industrial equipment. We apply the model for a European setting of one of their business areas. Due to confidentiality reasons all further specifications of company data have been omitted.

The questions to answer are the following: should refurbishment be promoted with or without the exchange option? At which locations are investments in facilities required and what are the appropriate capacity levels in these facilities? How high are the necessary inventory levels to support the service delivery strategy? Answering these questions also includes determining the routing of the parts through the network.

In order to keep the problem traceable, we aggregate all SKUs into one aggregate SKU $(C=1)$. The structure of the (potential) network is represented in Figure 5. The network contains two echelons with possible facility locations in five regions $(F=5)$ and the option to use an existing centralized distribution center in the second echelon. Moreover, customers who are served by traveling technicians are clustered into five customer locations corresponding to the five regions. Although traveling time (and cost) for these technicians are considered, we assume that ample capacity is available in the field. Furthermore, the transportation capacity for the products being transported between the different echelons of the network is assumed to be infinite as it is the responsibility of a third party logistics company. The two service delivery strategies are being offered across the network but prices differ between regions. As it is assumed that a remanufactured part performs as good as a new part, customers are not charged additionally when they receive a new part instead of a remanufactured one $\left(S P_{p r}=S P_{(p+P) r} \quad \forall p \in\{1, \ldots, P\}\right)$.

Figure 5: Case study network.

The five regions are characterized by different cost, demand and price structures. Clearly, it is difficult to estimate customers' sensitivity to pricing and marketing decisions of the company. Therefore, we search the optimal network structure and delivery strategy for different scenarios of prices and volumes. The volumes and prices are based on guidelines of the marketing department and an internal survey. As can be seen from Table 1, we allow for six demand levels ranging from a minimal market penetration (level 1) to full market potential (level 6). At the same time, the relative selling price of an exchange compared to a refurbishment alternates between three levels: low, intermediate and high.

Table 1 gives an overview of the results obtained for the different instances tested. We observe that for each volume and price setting, it is optimal to limit the network to a sole echelon, i.e. parts are transported directly form the customer to an all-round facility and from this facility back to a customer. The possibility to use a dedicated centralized distribution center is not selected by the model. The volume plays an important role in the level of centralization. Indeed, only two facilities should be opened at the lowest volume level, while the number of facilities can go up to 4 and even 5 facilities at the highest volume level. Nevertheless, we remark that the optimal profit level reduces only slightly when too few or too many facilities are opened. This relationship is graphically represented in Figure 6. Moreover, opening too 
Table 1: Summary results of case study.

many facilities is less detrimental than opening too few of them. Growing volume also justifies hiring more operators and the optimal utilization rates of the operators increases.

Figure 6: Relationship between profit and number of facilities.

As could be expected, the relative selling price of exchange service plays a key role to determine the optimal service delivery strategy. Whereas the refurbishment without exchange strategy is to be preferred at low price levels for exchange, regions will switch towards exchange service as the relative price of such service increases. If a high relative selling price can be obtained, regions should unanimously opt for an exchange strategy. At the intermediate relative price level, the refurbishment strategy should only be followed in one region. This particular region has the divergent feature that customers have a strong preference to recollect their own part and as such value refurbishment without exchange more strongly than customers in other regions. Furthermore, field technician costs are low in this region. Recall that refurbishment without exchange requires an additional visit of a technician compared to the exchange option. Both the lower wage cost of technicians and the higher willingness to pay for refurbishment explain the high attractiveness of a refurbishment strategy in this region. Interestingly, as the exchange service becomes more 
popular due to its increasing selling price, the optimal utilization rate of the operators goes down. Here the queueing dynamics are at play, lower utilization rates equal shorter lead times. Due to the higher inventory requirements of an exchange policy, shorter lead times are especially advantageous as some inventory holding costs can be avoided. In the exchange strategy, the potential savings in inventory advocate a higher investment in capacity and consequently target lower utilization rates in order to reduce the lead time of the remanufacturing process.

\section{Conclusions}

Although remanufacturing networks have been primarily studied in a military context, many companies have significant remanufacturing activities. Accordingly, in this paper we have taken a profit maximization perspective when optimizing the remanufacturing network design and delivery strategy. The choice of a certain service delivery strategy, either refurbishment with or without an exchange of the parts, has implications on the generated revenues and the cost parameters to optimize the network. Next to the service delivery decision and resulting profit perspective of the developed model, our contribution lies in the simultaneous solution of three related network design problems, i.e. the facility location, capacity and inventory problem.

Our research is motivated by a real-life case study at a worldwide manufacturer of industrial, construction and mining equipment. Within a multi-echelon repairable network structure, we determined the optimal number, type and location of remanufacturing facilities. Taking into account the stochastic nature of both demand and processing times, queuing theory was applied to set proper capacity and inventory levels, while upholding customer service. Due to the nonlinearity of the problem formulation, we solve the problem by applying a differential evolution search technique. The case study results emphasize the importance of volume as a driver for decentralization. Indeed, we observe that in the range of plausible volumes the number of remanufacturing facilities goes up from two to five as the volume increases. Secondly, the selling price is the main driver for the choice for the service delivery strategy. More interesting however, is the observation that the optimal network structure (capacity levels) is influenced by the choice of a service delivery strategy. As the optimal network structure differs between service delivery strategies, we emphasize the need to simultaneously analyze the network and service contract design.

The authors acknowledge that the insights retrieved from the case study need further backing by more case study research and sensitivity analysis. Nevertheless, our research shows that a holistic approach in network optimization is necessary. We also demonstrate that fundamental and applied research goes hand in hand. No need to say that the model offers several possibilities to extend the work such as the introduction of transportation batches, limited field technician capacity or non-Poisson distributed demand.

\section{A Correction factor $\phi$ in the GI/G/m-model}

$$
\phi\left(\rho_{f}, \tilde{c}_{I A_{f}}^{2}, \tilde{c}_{f}^{2}, s_{f}\right)= \begin{cases}\left(\frac{4\left(\tilde{c}_{I A_{f}}^{2}-\tilde{c}_{f}^{2}\right)}{4 \tilde{c}_{I A_{f}}^{2}-3 \tilde{c}_{f}^{2}}\right) \phi_{1}\left(s_{f}, \rho_{f}\right)+\left(\frac{\tilde{c}_{f}^{2}}{4 \tilde{c}_{I A_{f}}^{2}-3 \tilde{c}_{f}^{2}}\right) \psi\left(c^{2}, s_{f}, \rho_{f}\right) & \tilde{c}_{I A_{f}}^{2} \geq \tilde{c}_{f}^{2} \\ \left(\frac{\tilde{c}_{f}^{2}-\tilde{c}_{I A_{f}}^{2}}{2\left(\tilde{c}_{I A_{f}}^{2}+\tilde{c}_{f}^{2}\right)}\right) \phi_{3}\left(s_{f}, \rho_{f}\right)+\left(\frac{\tilde{c}_{f}^{2}+3 \tilde{c}_{I A_{f}}^{2}}{2\left(\tilde{c}_{I A_{f}}^{2}+\tilde{c}_{f}^{2}\right)}\right) \psi\left(c^{2}, s_{f}, \rho_{f}\right) & \tilde{c}_{I A_{f}}^{2} \leq \tilde{c}_{f}^{2}\end{cases}
$$

where 


$$
\begin{aligned}
& \phi_{1}\left(s_{f}, \rho_{f}\right)=1+\gamma\left(s_{f}, \rho_{f}\right) \\
& \phi_{3}\left(s_{f}, \rho_{f}\right)=\left(1-4 \gamma\left(s_{f}, \rho_{f}\right)\right) e^{-\frac{2\left(1-\rho_{f}\right)}{3 \rho_{f}}} \\
& \psi\left(c^{2}, s_{f}, \rho_{f}\right)= \begin{cases}1 & c^{2} \geq 1 \\
\phi_{4}\left(s_{f}, \rho_{f}\right)^{2\left(1-c^{2}\right)} & 0 \leq c^{2} \leq 1\end{cases}
\end{aligned}
$$

with

$$
\begin{aligned}
& \gamma\left(s_{f}, \rho_{f}\right)=\min \left\{0.24, \frac{\left(1-\rho_{f}\right)\left(s_{f}-1\right)\left[\left(4+5 s_{f}\right)^{0.5}-2\right]}{16 s_{f} \rho_{f}}\right\} \\
& c^{2}=\frac{\tilde{c}_{I A_{f}}^{2}+\tilde{c}_{f}^{2}}{2} \\
& \phi_{4}\left(s_{f}, \rho_{f}\right)=\min \left\{1, \frac{\phi_{1}\left(s_{f}, \rho_{f}\right)+\phi_{3}\left(s_{f}, \rho_{f}\right)}{2}\right\}
\end{aligned}
$$

\section{B Variance of the waiting time in the GI/G/m-model}

If $c_{W Q_{f}}^{2}$, the $\mathrm{SCV}$ of the waiting time in the queue at facility $f$, is known, its variance can be obtained according to

$$
\sigma_{W Q_{f}}^{2}=c_{W Q_{f}}^{2}\left(E W Q_{f}\right)^{2}
$$

Whitt (1993) proposes the following relationship for $c_{W Q_{f}}^{2}$, which is based on $c_{C W Q_{f}}^{2}$, the SCV of the conditional wait given that the facility $f$ is busy

$$
c_{W Q_{f}}^{2}=\left(c_{C W Q_{f}}^{2}+1-P\left(W Q_{f}>0\right)\right) / P\left(W Q_{f}>0\right)
$$

To calculate $c_{C W Q_{f}}^{2}$ the exact formula for the M/G/1 model is used as an approximation for the M/G/m and G/G/m model because the conditional delay should depend much more on the service time-distribution than on the interarrival time distribution

with

$$
c_{C W Q_{f}}^{2}=2 \rho_{f}-1+4\left(1-\rho_{f}\right) \tilde{d}_{f}^{3} / 3\left(\tilde{c}_{f}^{2}+1\right)^{2}
$$

$$
\tilde{d}_{f}^{3}= \begin{cases}3 \tilde{c}_{f}^{2}\left(1+\tilde{c}_{f}^{2}\right) & \text { if } \tilde{c}_{f}^{2} \geq 1 \\ \left(2 \tilde{c}_{f}^{2}+1\right)\left(1+\tilde{c}_{f}^{2}\right) & \text { if } \tilde{c}_{f}^{2}<1\end{cases}
$$

For the probability of delay, we have

$$
P\left(W Q_{f}>0\right)=P\left(W Q\left(\rho_{f}, \tilde{c}_{I A_{f}}^{2}, \tilde{c}_{f}^{2}, s_{f}\right)>0\right)=\min \{\kappa, 1\}
$$

where

$$
\kappa= \begin{cases}\kappa_{1} & \text { if } s_{f} \leq 6 \vee \gamma \leq 0.5 \vee \tilde{c}_{I A_{f}}^{2} \geq 1 \\ \kappa_{2} & \text { if } s_{f} \geq 7 \wedge \gamma \geq 1 \wedge \tilde{c}_{I A_{f}}^{2}<1 \\ \kappa_{3} & \text { if } s_{f} \geq 7 \wedge 0.5<\gamma<1 \wedge \tilde{c}_{I A_{f}}^{2}<1\end{cases}
$$

with

$$
\begin{aligned}
& \kappa_{1}=\rho_{f}^{2} \kappa_{4}+\left(1-\rho_{f}^{2}\right) \kappa_{5} \\
& \kappa_{2}=\tilde{c}_{I A_{f}}^{2} \kappa_{1}+\left(1-\tilde{c}_{I A_{f}}^{2}\right) \kappa_{6} \\
& \kappa_{3}=2\left(1-\tilde{c}_{I A_{f}}^{2}\right)(\gamma-0.5) \kappa_{2}+\left(1-2\left(1-\tilde{c}_{I A_{f}}^{2}\right)(\gamma-0.5)\right) \kappa_{1}
\end{aligned}
$$




$$
\begin{aligned}
& \kappa_{4}=\min \left\{1, \frac{1-z\left[\frac{\left.\left(1+\tilde{c}_{f}^{2}\right)\left(1-\rho_{f}\right){\sqrt{s_{f}}}^{\tilde{c}_{I A_{f}}+\tilde{c}_{f}^{2}}\right]}{1-z\left[\left(1-\rho_{f}\right) \sqrt{s_{f}}\right]} P\left(W Q(M / M / m)_{f}>0\right)\right\}}{\kappa_{5}=\min \left\{1, \frac{1-z\left[\frac{2\left(1-\rho_{f}\right) \sqrt{s}_{f}}{\tilde{\tilde{c}}_{I A_{f}}^{2}+1}\right]}{1-z\left[\left(1-\rho_{f}\right) \sqrt{s_{f}}\right]} P\left(W Q(M / M / m)_{f}>0\right)\right\}}\right. \\
& \kappa_{6}=1-z[\gamma] \\
& \gamma=\left(s_{f}-s_{f} \rho_{f}-0.5\right) / \sqrt{s_{f} \rho_{f}\left(\tilde{c}_{I A_{f}}^{2}+\tilde{c}_{f}^{2}\right) /\left(1+\tilde{c}_{f}^{2}\right)} \\
& P\left(W Q(M / M / m)_{f}>0\right)=\left(\left(s_{f} \rho_{f}\right)^{s_{f}}\right) /\left(s_{f} !\left(1-\rho_{f}\right)\right) \zeta \\
& \zeta \equiv\left(\frac{\left(s_{f} \rho_{f}\right)_{f}}{s_{f} !\left(1-\rho_{f}\right)}+\sum_{k=0}^{s_{f}-1} \frac{\left(s_{f} \rho_{f}\right)^{k}}{k !}\right)^{-1}
\end{aligned}
$$

and $z[x]$ the standard normal cumulative distribution function.

\section{Acknowledgment}

This work is supported by the Atlas Copco Research Chair in Service Systems and by the research project G.0333.10N of the Research Fund Flanders (FWO) (Belgium).

\section{References}

Adjiman, C., I. Androulakis, C. Floudas, 1997. Global optimization of minlp problems in process synthesis and design. Computers and Chemical Engineering, 21, S445-S450.

Androulakis, I., C. Maranas, C. Floudas, B. Alpha, 1995. A global optimization method for general constrained nonconvex problems. Journal of Global Optimization, 104, 201-217.

Babu, B., R. Angira, 2002. A differential evolution approach for global optimization of MINLP problems. Proceedings of 4th Asia-Pacific Conference on Simulated Evolution And Learning, 2, 880-884.

Buzacott, J., J. Shanthikumar. Stochastic Models of Manufacturing Systems. Prentice Hall, Englewood Cliffs, NY, 1993.

Caggiano, K. E., J. A. Muckstadt, J. A. Rappold, 2006. Integrated real-time capacity and inventory allocation for reparable service parts in a two-echelon supply system. M\&Som-Manufacturing \& Service Operations Management, 8 (3), 292-319.

Chelouah, R., P. Siarry, 2000. Tabu search applied to global optimization. European Journal of Operational Research, 123, $256-270$.

Cohen, M. A., N. Agrawal, V. Agrawal, 2006. Winning in the aftermarket. Harvard Business Review, 84 (5), $129-138$.

Daskin, M. S., C. R. Coullard, Z. J. M. Shen, 2002. An inventory-location model: Formulation, solution algorithm and computational results. Annals of Operations Research, 110 (1-4), 83-106.

Diaz, A., M. C. Fu, 1997. Models for multi-echelon repairable item inventory systems with limited repair capacity. European Journal of Operational Research, 97, 480-492.

Ferguson, M. E., L. B. Toktay, 2006. The effect of competition on recovery strategies. Production and Operations Management, 15 (3), 351-368.

Fonseca, M. C., A. Garcia-Sanchez, M. Ortega-Mier, F. Saldanha-da Gama, 2010. A stochastic bi-objective location model for strategic reverse logistics. Top, 18, 158-184.

Graves, S. C., 1985. A multi-echelon inventory model for a repairable item with one-for-one replenishment. Management Science, 31 (10), 1247-1256.

Gross, D., D. R. Miller, R. M. Soland, 1983. A closed queuing network model for multi-echelon repairable item provisioning. Iie Transactions, 15 (4), 344-352. 
Hopp, W., M. Spearman. Factory Physics. The McGraw-Hill Companies, New York, 2000.

Jiang, C. Z., F. Xu, Z. H. Sheng, 2010. Pricing strategy in a dual-channel and remanufacturing supply chain system. International Journal of Systems Science, 41 (7), 909-921.

Kesavan, P., P. Barton, 2000. Generalized branch-and-cut framework for mixed-integer nonlinear optimization problems. Computers and Chemical Engineering, 24, 1361-1366.

Lambrecht, M., P. Ivens, N. Vandaele, 1998. ACLIPS: A capacity and lead time integrated procedure for scheduling. Management Science, 44 (11), 1548-1561.

Lampinen, J., I. Zelinka. Mechanical Engineering Design Optimization by Differential Evolution, chapter 8, 127-146. McGrawHill, London, 1999.

Lieckens, K., N. Vandaele, 2007. Reverse logistics network design: The extension towards uncertainty. Computers and Operations Research, 34 (2), 395 - 416.

Mak, H. Y., Z. J. M. Shen, 2009. A two-echelon inventory-location problem with service considerations. Naval Research Logistics, 56 (8), 730-744.

Mathur, M., S. Karale, S. Priye, V. Jayaraman, B. Kulkarni, 2000. Ant colony approach to continuous function optimization. Industrial and Engineering Chemistry Research, 39, 3814-3822.

Melo, M. T., S. Nickel, F. Saldanha-da Gama, 2009. Facility location and supply chain management - a review. European Journal of Operational Research, 196 (2), 401-412.

Muckstadt, J., 1973. Model for a multi-item, multi-echelon, multi-indenture inventory system. Management Science Series BApplication, 20 (4), 472-481.

Nozick, L. K., M. A. Turnquist, 2001. A two-echelon inventory allocation and distribution center location analysis. Transportation Research Part E-Logistics and Transportation Review, 37 (6), 425-441.

Pawar, K. S., A. Beltagui, J. Riedel, 2009. The pso triangle: designing product, service and organisation to create value. International Journal of Operations \& Production Management, 29 (5), 468-493.

Rajasekaran, S., 2000. On simulated annealing and nested annealing. Journal of Global Optimization, 16, 43-56.

Rappold, J. A., B. D. Van Roo, 2009. Designing multi-echelon service parts networks with finite repair capacity. European Journal of Operational Research, 199 (3), 781-792.

Ryoo, H., N. Sahinidis, 1995. Global optimization of nonconvex nlps and minlps with applications in process design. Computers and Chemical Engineering, 19 (5), 551-566.

Ryoo, H., N. Sahinidis, 1996. A branch-and-reduce approach to global optimization. Journal of Global Optimization, 8 (2), 107-138.

Savaskan, R. C., S. Bhattacharya, L. N. Van Wassenhove, 2004. Closed-loop supply chain models with product remanufacturing. Management Science, 50 (2), 239-252.

Shanthikumar, J., J. Buzacott, 1981. Open queueing network models of dynamic job shops. International Journal of Production Research, 19 (3), 255-266.

Shen, Z. J. M., C. Coullard, M. S. Daskin, 2003. A joint location-inventory model. Transportation Science, 37 (1), $40-55$.

Sherbrooke, C. C., 1986. Vari-metric - improved approximations for multi-indenture, multiechelon availability models. Operations Research, 34, 311-319.

Sleptchenko, A., M. C. van der Heijden, A. van Harten, 2003. Trade-off between inventory and repair capacity in spare part networks. Journal of the Operational Research Society, 54 (3), 263-272.

Storn, R., K. Price, 1997. Differential evolution - a simple end efficient heuristic for global optimization over continuous spaces. Journal of Global Optimization, 11, 341-359.

Tadikamalla, P., 1984. A comparison of serveral approximations to the lead time demand distribution. Omega International Journal of Management Science, 12, 575-581.

Vaidyanathan, R., M. El-Halwagi. Global Optimization of Nonconvex Minlps by Interval Analysis, 175-193. Kluwer Academic Publishers, Dordrecht, 1996.

van Ommeren, J. C. W., A. F. Bumb, A. V. Sleptchenko, 2006. Locating repair shops in a stochastic environment. Computers \& Operations Research, 33, 1575-1594.

Vorasayan, J., S. M. Ryan, 2006. Optimal price and quantity of refurbished products. Production and Operations Management, 15, 369-383. 
Wah, W., T. Wang, 1999. Efficient and adaptive lagrange-multiplier methods for nonlinear continuous global optimization. Journal of Global Optimization, 14, 1-25.

Westerlund, T., H. Skrifvars, I. Harjunkoski, R. Pörn, 1998. An extended cutting plane method for a class of non-convex minlp problems. Computers and Chemical Engineering, 22 (3), 357-365.

Whitt, W., 1983. The queueing network analyzer. The Bell System Technical Journal, 62 (9), 2779-2815.

Whitt, W., 1993. Approximations for the GI/G/m queue. Production and Operations Management, 2 (2), $114-161$.

Zuo-Jun Max, S., M. S. Daskin, 2005. Trade-offs between customer service and cost in integrated supply chain design. Manufacturing \& Service Operations Management, 7 (3), 188-207. 